\title{
Effects of Dipole Magnetic Fields on Axisymmetric p-Mode Pulsations
}

\author{
Hideyuki Saio \\ Astronomical Institute, Graduate School of Science, Tohoku University, \\ Sendai, Japan \\ Alfred Gautschy \\ ETH-Library, Rämistrasse 101, 8092 Zürich, Switzerland
}

We investigated nonradial pulsations in the presence of a dipole magnetic field in a non-rotating $1.7 M_{\odot}$ ZAMS star. Formally, like in the case of pulsationrotation coupling (Lee \& Saio, 1986), the angular dependence of the pulsations is expanded into a series of spherical harmonics of different latitudinal degrees $\ell$. To start with, we considered only axisymmetric $(m=0)$ modes under the adiabatic and the Cowling approximations. In contrast to previous studies of pulsationmagnetic field interaction (Dziembowski \& Goode, 1996; Bigot et al., 2000; Cunha \& Gough, 2000), we retained the latitudinal derivatives of the perturbed quantities.

The magnetic perturbation variables oscillate rapidly in space when the magnetic pressure is small compared to the gas pressure. These rapid oscillations are caused by inwardly propagating magnetic slow waves which should be dissipated before they reach the stellar center. Therefore, the presence of slow waves in the problem damp pulsations (Roberts \& Soward, 1983), even in the adiabatic case. To account for this effect we imposed running-wave conditions on the magnetic perturbations at a depth where the gas pressure dominates over magnetic pressure (e.g., at $r / R \simeq 0.83$ at $B_{p}=1 \mathrm{kG}$ for the model presented here).

Fig. 1 shows some preliminary results. The left panel of Fig. 1 contains oscillation frequencies of odd modes as a function of the magnetic field strength at the magnetic pole, $B p$. Only $\ell=1$ (open squares) and $\ell=3$ modes (crosses) are shown. Numerous and complicated avoided crossings are seen to develop as the field strength increases. The right panel of Fig. 1 shows, for the case of a kiloGauss field, that the damping rate and the frequency shift $(\Delta \nu=\nu(B p)-$ $\nu(B p=0))$ of $\ell=1$ modes are large close to the crossings. The magnitudes of both $\Delta \nu$ and $\omega_{i} / \omega_{r}$ vary cyclically as a function of the oscillation frequency. The eigenfrequency behaviour seen in Fig. 2 shows that our results for $\ell=1$ agree qualitatively with those obtained by Cunha \& Gough (2000) using a variational principle. 

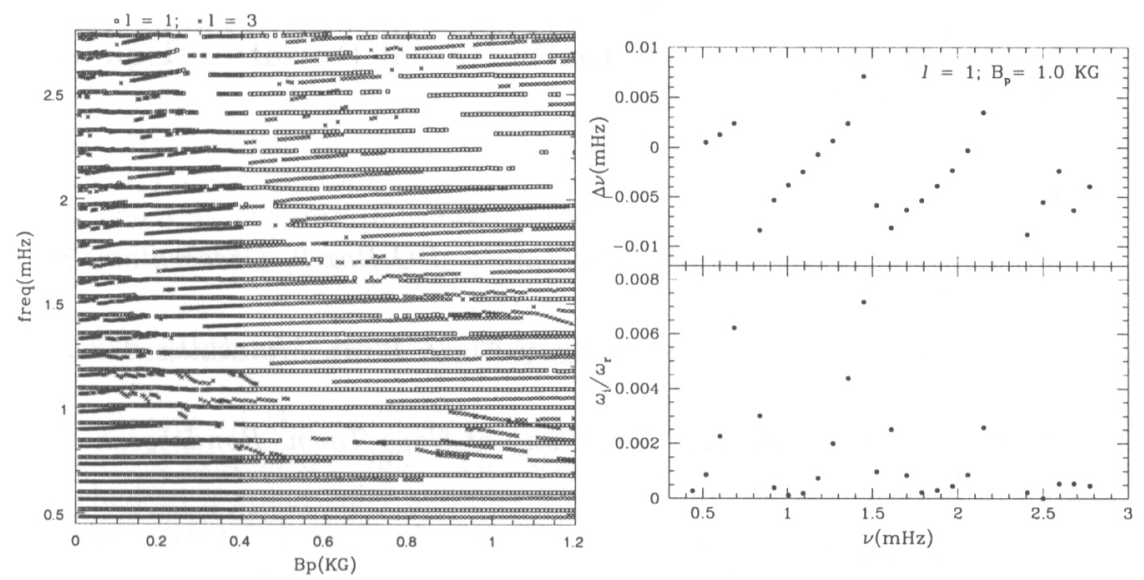

Figure 1. Left: oscillation frequencies of odd modes as a function of $B p$; right: frequency shifts and damping rates for $B_{p}=1 \mathrm{kG}$.

\section{References}

Bigot, L., Provost, J., Berthomieu, G., Dziembowski, W.A., \& Goode, P.R. 2000, A\&A, 356, 218

Cunha, M.S. \& Gough, D. 2000, MNRAS, 319, 1020

Dziembowski, W.A. \& Goode, P.R. 1996, ApJ, 458, 338

Lee, U. \& Saio, H. 1986, MNRAS, 221, 365

Roberts, P.H. \& Soward, A. 1983, MNRAS, 205, 1171 